\title{
UTILISATION DE POUDRES ET D'EXTRAITS TOTAUX ISSUS DE PLANTES LOCALES DES GENRES Ocimum sp. ET Mentha sp. COMME BIOPESTICIDES DANS LA LUTTE CONTRE Callosobruchus maculatus FAB.
}

\author{
F. JOHNSON, B. SERI-KOUASSI, L.R.N.ABOUA, K. FOUA-BI
}

Laboratoire de Zoologie-Biologie Animale, UFR Biosciences, Université de Cocody, 22 BP 582 Abidjan, Côte d'Ivoire

\section{RESUME}

Quatre espèces végétales de la famille des Labiaceae (Ocimum canum L., O. gratissimum L., Mentha spicata L. et M. piperita L.) ont été testées pour leur effet insecticide sur Callasobruchus maculatus FAB. Les poudres et les extraits totaux de ces plantes ont réduit de façon significative l'infestation, la durée de vie, la fécondité et l'émergence de l'insecte dans les stocks. Les résultats obtenus avec les extraits totaux ont été plus significatifs que ceux des poudres. Ainsi, l'extrait total de $M$. piperita s'est avéré plus efficace que ceux de O. canum, $O$. gratissimum, et $M$. spicata. L'application de $40 \mu \mathrm{l}$ d'extrait pour $10 \mathrm{~g}$ de niébé (une dose de $4 \mu \mathrm{l} / \mathrm{g}$ ), sur 30 couples de $C$. maculatus, a réduit la fécondité, le taux d'infestation et le taux d'émergence de l'insecte respectivement de 99,67\%; $98 \%$; et 91,98\%. Quant à la durée de vie, elle est passée dans les mêmes conditions de $5,8 \pm 1,5$ à $1,2 \pm 0,4 \mathrm{j}$; soit une réduction de $79,34 \%$. Par ailleurs, le taux d'émergence enregistré après le traitement des œufs par ces différents extraits a été quasiment nul. Contrairement aux œufs, les larves et les nymphes n'ont pas été sensibles à ces traitements.

Mots clés : Biopesticides, Callasobruchus maculatus, traitement, Mentha, Ocimum, Côte d'Ivoire.

\author{
ABSTRACT \\ USE OF POWDER AND TOTAL EXTRACT OF LOCAL PLANTS FROM THE GENERA Ocimum sp. AND Mentha sp. AS \\ BIOPESTICIDES IN THE CONTROL OF Callosobruchus maculatus FAB.
}

Four plant species, Ocimum canum L., O. gratissimum L., Mentha spicata L. and M. piperita L. (Labiaceae) were tested to assess their insecticidal effects on Callasobruchus maculatus. Results showed that these plants reduced significantly infestation rates of stored cowpea seeds. They reduced the lifespan, fecundity, and adult emergence rate of $\mathrm{C}$. maculatus. Total extracts were more efficient than the powders. M. piperita exhibited the most important activity. When applied as a total extract, the plant produced better results. Indeed, the application of $40 \mu \mathrm{l}$ of extract $(4 \mu \mathrm{l} / \mathrm{g})$ reduced fecundity, infestation rates and insect emergence by 99, $67 \%$; $98 \%$ and 91, $98 \%$, respectively. The lifespan decreased from $5.8 \pm 1.5$ to $1.2 \pm 0.4$ days. No significant emergence was recorded after the eggs of $\mathrm{C}$. maculatus have been treated. The recorded activity of these plants was due to their ovicidal effect. Nevertheless, the larvae and the pupa did not show any sensitivity to the treatment.

Key words : Biopesticides, Callasobruchus maculatus, treatment, Mentha, Ocimum, Côte d'Ivoire. 


\section{INTRODUCTION}

Le niébé (Vigna unguiculata (L) WALP) est une légumineuse à vertus nutritionnelles et fertilisantes du sol. C'est donc une plante de très grande valeur économique, pour les pays en voie de développement, et par conséquent recommandée aux populations pour corriger les déséquilibres alimentaires. Sa teneur en protéines (22 à $25 \%$ ) est 2 à 3 fois plus élevée que celle des céréales (Stanton, 1970). Cette richesse en protéines lui a valu le nom de " viande de pauvres ». En outre, elle constitue une source appréciable d'énergie, d'éléments nutritifs et de vitamines (Rachie et Robert, 1974). Cependant, cette plante fait l'objet d'attaques par plusieurs insectes, aussi bien au champ que dans les stocks. Parmi ces ravageurs, les Coléoptères et les Lépidoptères sont le plus souvent rencontrés (Kossou et Aho, 1993). Callosobruchus maculatus FAB (Coléoptère, Bruchidae) prend une part très active dans la déprédation des graines de niébé en Côte d'Ivoire, principalement dans la ville d'Abidjan (Doumbia, 1992 ; Aboua, 1997). Ces ravageurs détruisent d'énormes quantités de produits stockés. Ils infestent les graines depuis les champs à un taux faible (Caswell, 1961). Mais, c'est au cours du stockage que la denrée est plus endommagée. La destruction peut affecter 60 à $80 \%$ des graines entreposées au bout de 6 mois si aucune mesure de protection phytosanitaire n'est prise (Nuto et Glitho, 1990). En Côte d'Ivoire, l'infestation des gousses de niébé par les bruches, provoquant la perte des récoltes, a entraîné la négligence de cette denrée par certains paysans au profit d'autres cultures vivrières. La disparition de cette culture pourrait causer de graves préjudices aux populations de plusieurs pays africains, compte tenu de ses valeurs nutritives (Seri-Kouassi, 2004). II apparaît donc nécessaire d'engager une lutte contre les déprédateurs des cultures et des graines de niébé en stock. Ainsi, plusieurs méthodes de lutte ont-elles été élaborées. Certaines d'entre elles, (notamment la lutte chimique), quoique efficaces, présentent des dangers pour l'homme et son environnement à cause de la toxicité des produits utilisés (Thiam et Ducom, 1993). Par contre, les substances naturelles sont moins toxiques pour l'homme que les insecticides de synthèse (Ostermann, 1993). Ainsi, Foua-Bi (1993) a rapporté que les paysans africains utilisaient jadis des substances naturelles pour protéger leurs récoltes contre les insectes.
Quelques travaux portant sur les insecticides naturels ont été réalisés au Niger (Alzouma et Boubacar, 1987) et au Burkina-Faso (Dabiré, 1993). En Côte d'Ivoire, très peu de travaux ont été entrepris dans ce domaine. Gakuru et FouaBi (1995) et Seri-Kouassi (2005) ont utilisé avec succès des extraits de plantes pour lutter contre les insectes et les moisissures.

La présente étude a donc pour but de tester, au laboratoire, les effets des poudres et des extraits totaux de quatre plantes locales sur certains paramètres biologiques de Callosobruchus maculatus en vue de réduire les dégâts causés par cette bruche aux graines de niébé.

\section{MATERIEL ET METHODES}

\section{MATERIEL}

Le matériel animal a été constitué des adultes de l'insecte Callosobruchus maculatus FAB. Les individus de cette espèces ont été récoltés par tamisage des graines de niébé, provenant d'un champ d'expérimentation installé à la ferme de l'Université d'AboboAdjamé ; ces graines ayant été stockées dans des conditions ambiantes. $\left(T=25,7 \pm 0,8^{\circ} \mathrm{C}\right.$, HR 89,7 $\pm 3,6 \%$ ).

Le matériel végétal a été constitué d'une part, de la plante hôte, le niébé (Vigna unguiculata L. WALP) (Papilionaceae) et d'autre part, de plantes aromatiques appartenant à la famille des Labiaceae, dont deux espèces du genre Ocimum (O. gratissimum $L$. et $O$. canum $L$. et deux autres du genre Mentha (Mentha piperita L. et M. spicata L.).

Une broyeuse électrique a servi à moudre les feuilles séchées utilisées pour le traitement. Les pesées ont été faites sur une balance de marque SARTORIUS (précision $1 \mu \mathrm{g}$ ).

\section{METHODES}

Les extraits totaux ont été obtenus par macération des produits de la mouture des feuilles après séchage. Ainsi, $300 \mathrm{~g}$ de poudre de chaque espèce végétale ont été introduits dans un flacon Erlen-meyer auxquelles ont été ajoutés 2 litres d'alcool éthylique à $90^{\circ}$. Le mélange a été soumis à une agitation mécanique (à froid), puis macéré pendant $48 \mathrm{~h}$, puis filtré à l'aide de papier whatman $\mathrm{N}^{\circ} 2$. 
La solution obtenue a été passée à l'évaporateur rotatif en vue d'éliminer l'alcool ayant servi à l'extraction. Les traitements ont été réalisés à l'aide de poudres et d'extraits totaux de plantes, avant et après infestation. Le traitement effectué avant infestation par C. maculatus a permis de rechercher l'effet insecticide ou insectifuge des plantes sur l'insecte adulte. Le traitement après infestation par C. maculatus, a eu pour but de rechercher les effets de ces plantes sur les œufs, les larves et les nymphes.

Pour tester l'effet insecticide ou insectifuge des plantes sur l'insecte adulte, 100 graines de niébé pesant au total $10 \mathrm{~g}$, placées dans chacune des 5 boîtes de Pétri d'essais ont reçu respectivement $0,100,200,300$ et $400 \mathrm{mg}$ de poudre, soit $0,10,20,30$ et $40 \mathrm{mg}$ de poudre / $\mathrm{g}$ de graines de niébé. Le contenu de chaque boîte a été mélangé, de sorte à recouvrir toutes les graines par la poudre. Dans les mêmes conditions, les graines ont reçu des quantités croissantes d'extraits de plante : soit $0,10,20$, 30 et $40 \mu \mathrm{l}$ d'extraits correspondant à $0,1,2,3$ et $4 \mu \mathrm{l}$ d'extraits $/ g$ de niébé. Les boîtes de Pétri ont ensuite été agitées en vue d'enrober toutes les graines de produit. Ensuite, 10 couples de $C$. maculatus nouvellement émergés ont été introduits dans chaque boîte de Pétri. Les couples ont régulièrement été suivis jusqu'à la mort des insectes. Les paramètres biologiques déterminés ont été le taux d'infestation (nombre de graines infestées sur le nombre total de graines, exprimé en \%), la durée moyenne de vie (période moyenne comprise entre l'émergence des insecte et leur mort), la fécondité (nombre moyen d'œufs pondus par femelle), et le taux d'émergence (nombre d'insectes adultes émergés sur le nombre d'œufs pondus exprimé en \%).

Pour rechercher l'effet ovicide des plantes sur les formes cachées de l'insecte, le traitement a été appliqué aux graines portant des œufs âgés de 0, 1, 2 et 3 j. Quant à l'effet larvicide, il a été testé en appliquant le traitement aux graines portant des larves à différents stades de développement ; les différents âges étant déterminés à partir de la ponte des œufs par les femelles : L1 (œufs âgés de 4 à 6 j), L2 (œufs de 7 à 9 j), L3 (10 à 15 j après ponte), L4 ou prénymphes (16 à 19 j après ponte) nymphes (plus de $20 \mathrm{j}$ après ponte) (Aboua, 1997). Toutes ces opérations ont été répétées 3 fois.

Deux autres paramètres, la DL50 (la dose de produit capable de causer $50 \%$ de mort au bout de $24 \mathrm{~h}$ ) et le TL50 (le temps au bout duquel $50 \%$ des insectes meurent à la suite de l'application des différents produits) ont été déterminés en vue d'évaluer l'efficacité des traitements appliqués.

Le test de Khi-deux de Person au seuil de $5 \%$ a permis à comparer l'effet des différents traitements sur la durée moyenne de vie des insectes adultes. L'analyse de variances (ANOVA), suivie du test de Student-NewmanKeuls au seuil de $5 \%$ a servi à comparer les effets des poudres et des extraits totaux des 4 espèces végétales aux différentes doses d'une part et ceux des 2 types de traitement. Le logiciel statistica 6.0 a été utilisé pour les analyses statistiques.

\section{RESULTATS}

\section{TRAITEMENT DES GRAINES AVANT INFESTATION}

\section{Effet des traitements sur la durée de vie de l'adulte de $C$. maculatus}

Chez les témoins, les premiers insectes morts ont été enregistrés à partir du $3^{\mathrm{e}} \mathrm{j}$ après émergence et les derniers au $8^{\mathrm{e}} \mathrm{j}$ (Figures 1 à 4). Sur les graines traitées avec les différentes doses de poudres et d'extrait des 4 plantes, les insectes morts ont été observés à partir du $1^{\mathrm{er}} \mathrm{j}$ de traitement. II s'en est suivi une évolution progressive de la mortalité allant de 1 à 5 j pour les poudres des 4 espèces végétales (toutes doses confondues) et pour les extraits de $M$. spicata et $M$. piperita aux doses de 1 et $2 \mu \mathrm{l} / \mathrm{g}$. La mortalité s'est étendue sur $3 \mathrm{j}$, pour les doses de 3 et $4 \mu \mathrm{l} / \mathrm{g}$ avec $M$. spicata et $M$. piperita. La totalité des insectes traités avec $M$. piperata à la dose de $4 \mu \mathrm{l} / \mathrm{g}$ étaient morts dès le $2^{\mathrm{e}} \mathrm{j}$. Les extraits totaux ont donc semblé plus efficaces que les poudres. 

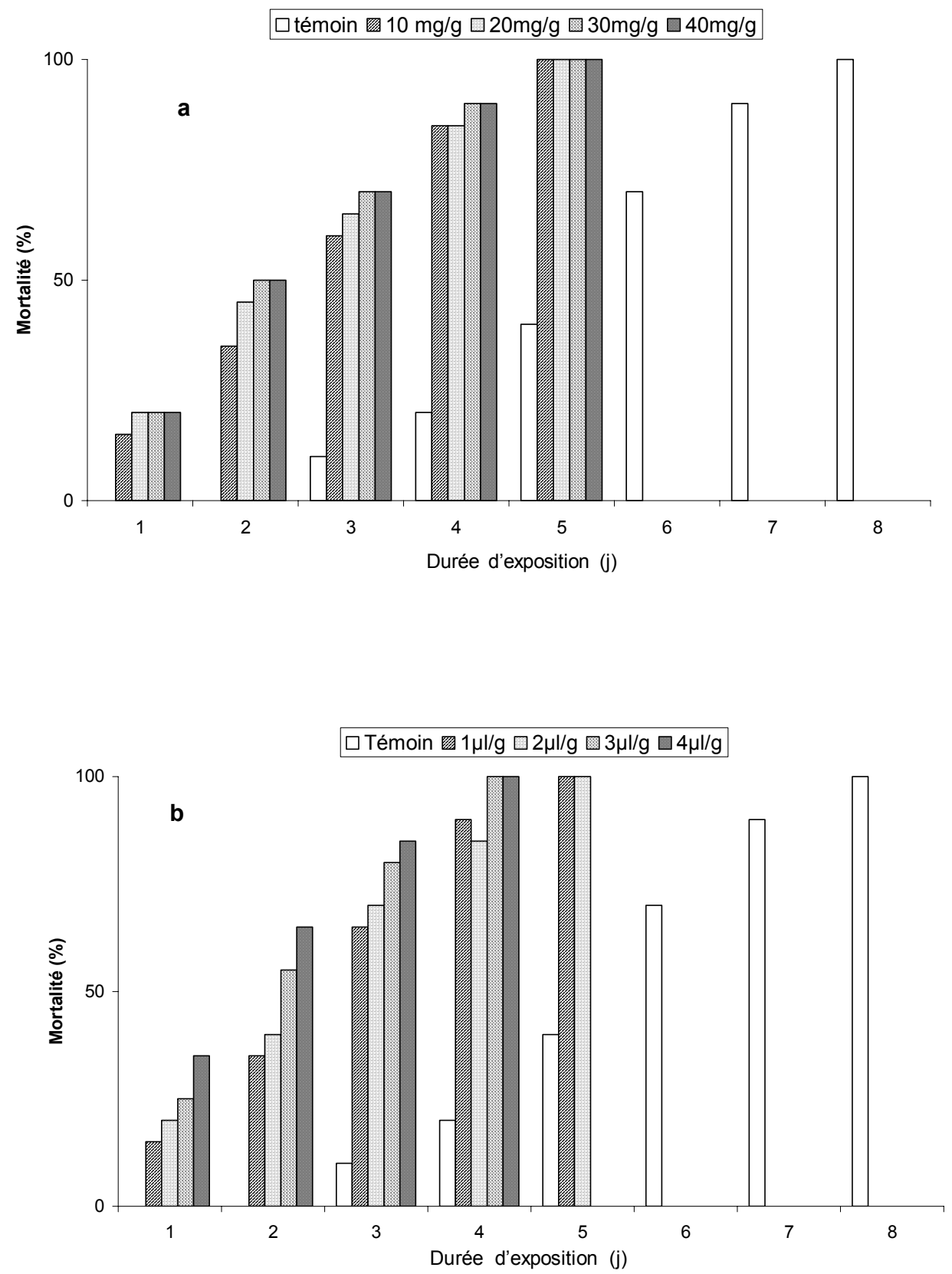

Figure 1 : Effets de la poudre (a) et de l'extrait total (b) de Ocimum gratissimum sur la mortalité de Callosobruchus maculatus.

Effects of powder (a) and total extracts (b) of Ocimum gratissimum on the mortality of Callosobruchus maculatus. 

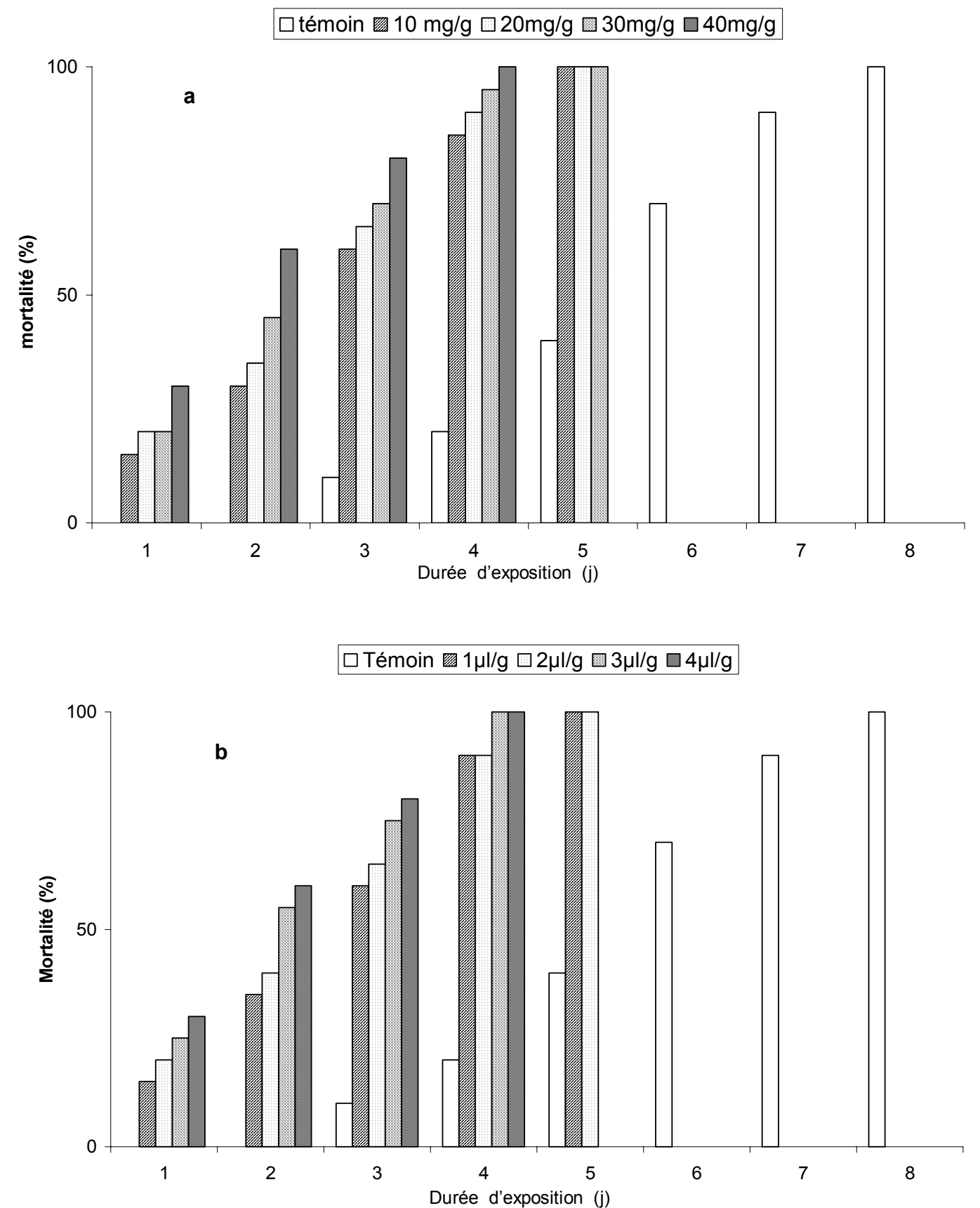

Figure 2 : Effets de la poudre (a) et de l'extrait total (b) de Ocimum canum sur la mortalité de Callosobruchus maculatus.

Effects of powder (a) and total extracts (b) of Ocimum canum on the mortality of Callosobruchus maculatus. 

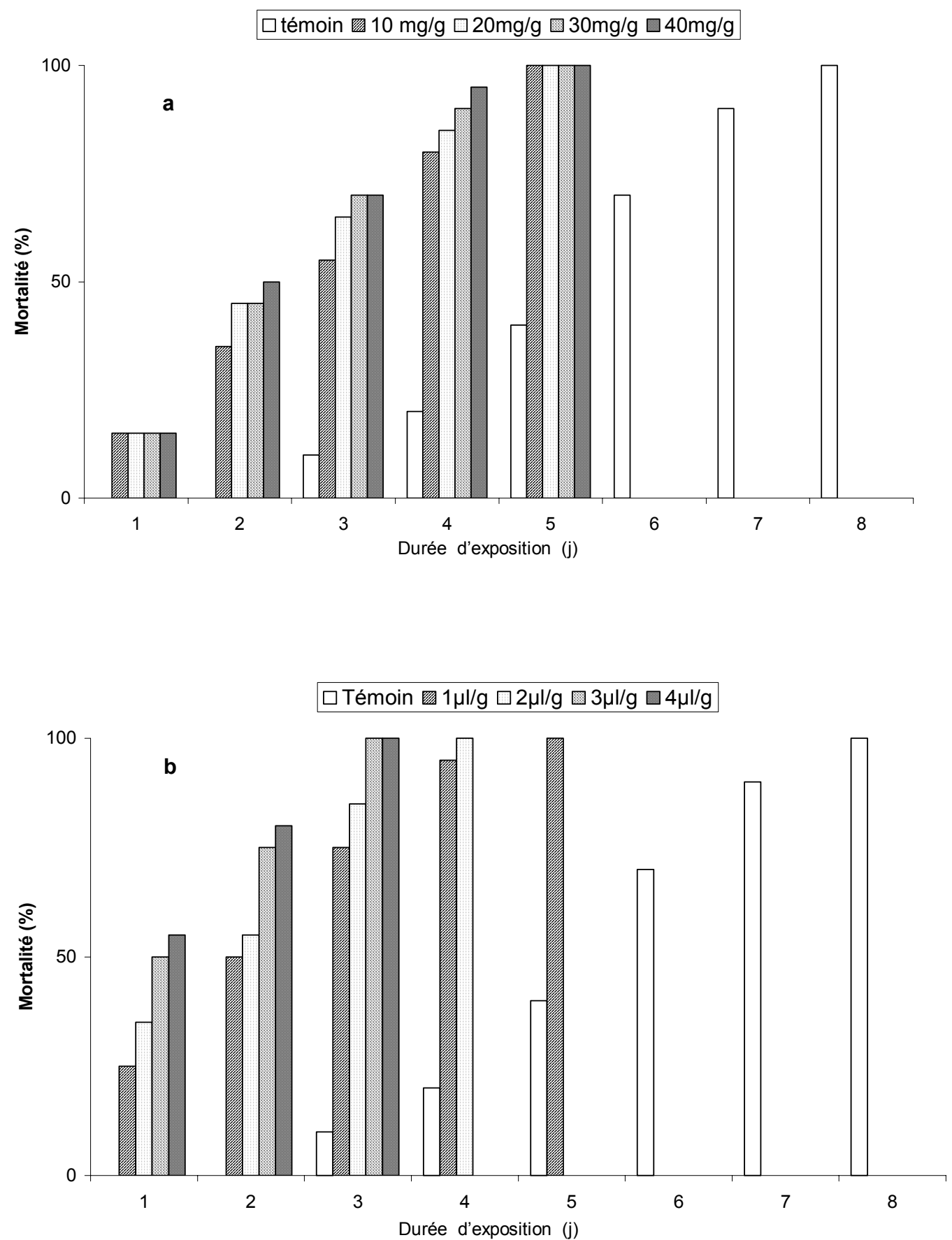

Figure 3 : Effets de la poudre (a) et de l'extrait total (b) de Mentha spicata sur la mortalité de Callosobruchus maculatus.

Effects of powder (a) and total extracts (b) of Mentha spicata on the mortality of Callosobruchus maculatus. 

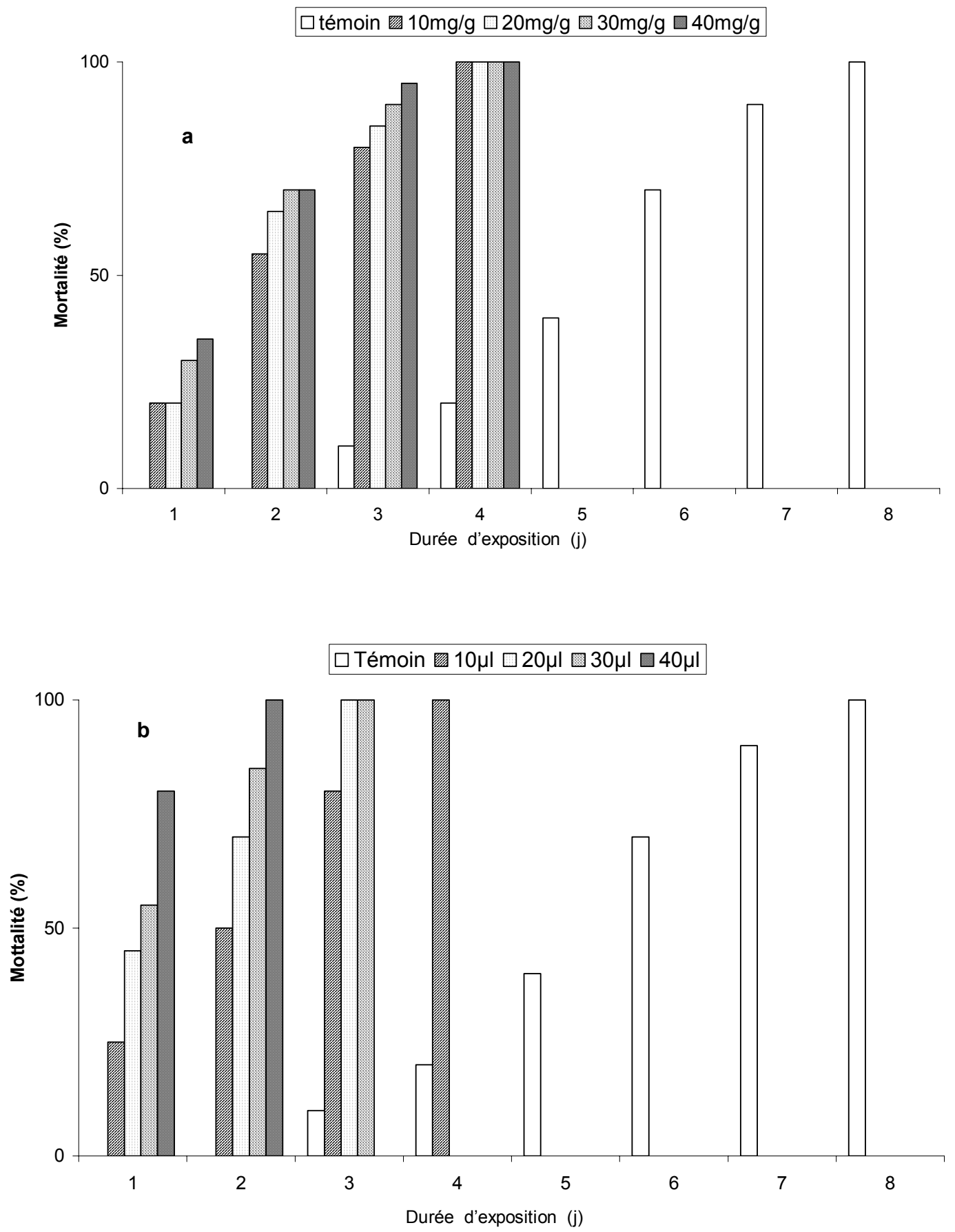

Figure 4 : Effets de la poudre (a) et de l'extrait total (b) de Mentha piperita sur la mortalité de Callosobruchus maculatus.

Effects of powder (a) and total extracts (b) of Mentha piperita on the mortality of Callosobruchus maculatus. 
Détermination de la dose létale 50 (DL50)

Les doses létales ont été atteintes pour les traitements avec les extraits de $M$. spicata et de $M$. piperita aux doses respectives de $3 \mu \mathrm{l} / \mathrm{g}$ et $2,6 \mu \mathrm{l} / \mathrm{g}$. Les autres plantes n'ont entraîné la mort de $50 \%$ des insectes qu'au-delà de $24 \mathrm{~h}$ après le traitement (Figure 5).

\section{Détermination du temps létal 50 (TL50)}

Les TL50 déterminés à partir de $40 \mu$ d'extrait des différentes plantes ont été très faibles, ils ont varié entre $0,5 \mathrm{j}$ pour $M$. piperata et 1,7 j pour $O$. canum (Tableau 1). Par contre ceux enregistrés lorsque les doses de poudre ont été appliquées sont relativement plus élevés. A la dose de $40 \mathrm{mg} / \mathrm{g}$, les différentes poudres ont présenté des TL50 compris entre à 1,5 jours pour $M$. piperita et 2,2 jours pour O. canum.

\section{Effet des traitements sur le taux d'infestation}

La valeur du taux d'infestation obtenue avec le témoin a été de $100 \%$. Pour les quatre espèces de plante et à toutes les doses, le traitement avec les poudres a permis d'obtenir des taux d'infestations élevés, supérieurs à $50 \%$ (Tableau 2). Lorsque les extraits totaux sont appliqués, les valeurs du taux d'infestation ont été également importants à la dose de $1 \mu \mathrm{l} / \mathrm{g}$ pour les quatre plantes et à celle de $2 \mu \mathrm{l} / \mathrm{g}$ pour les espèces du genre Ocimum. A cette dernière dose, l'infestation a considérablement baissé pour atteindre respectivement les taux de $48 \pm 2,16 \%$ et $37 \pm 2,16 \%$ pour $M$. spicata et M. piperita. Cette diminution a été encore plus remarquable aux doses de 3 et $4 \mu \mathrm{l} / \mathrm{g}$ pour toutes les plantes; la plus faible valeur $(2 \pm 2,8 \%)$ étant obtenue avec $M$. piperita.
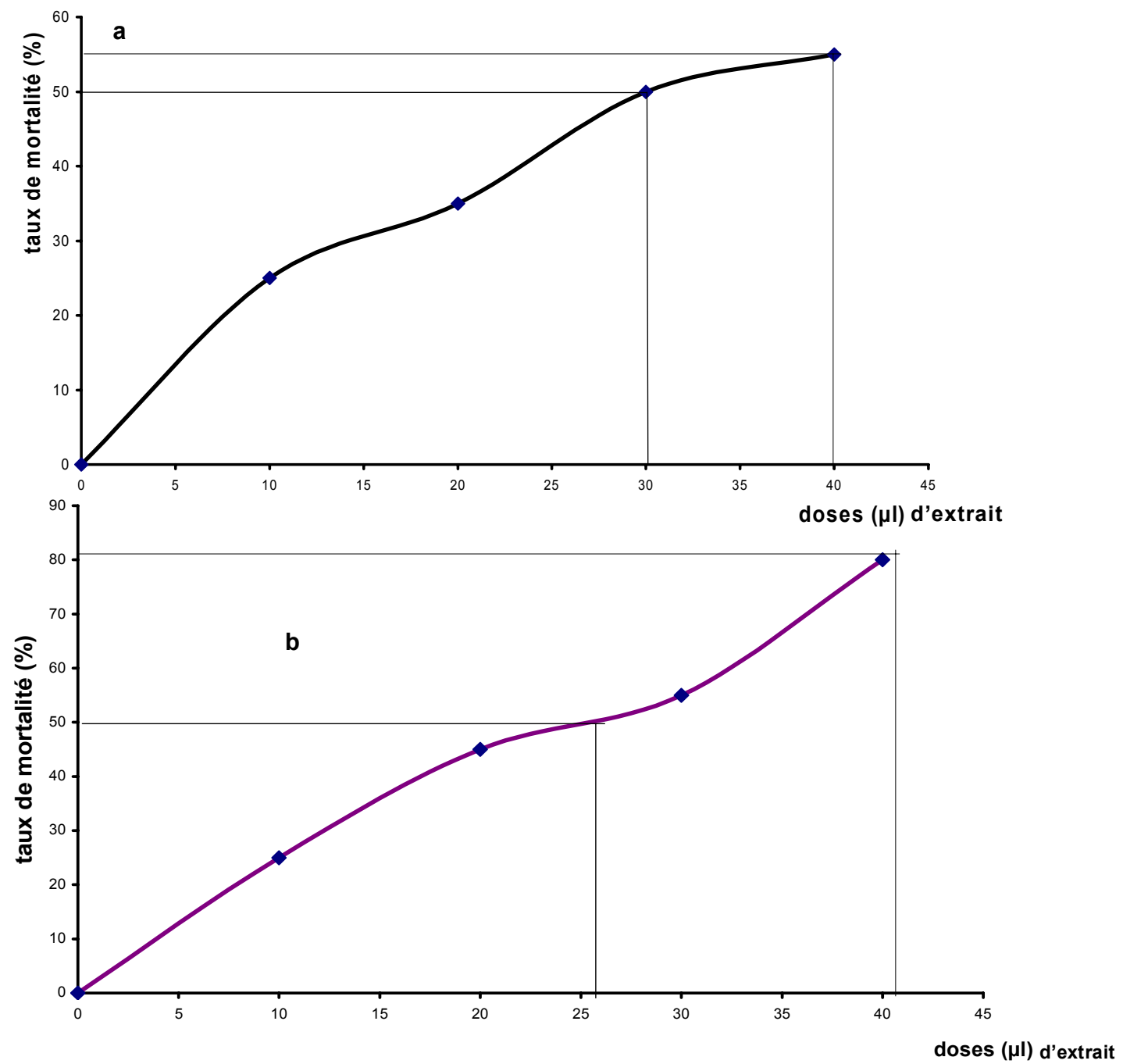

Figure 5 : Mortalité de Callosobruchus maculatus en fonction des doses d'extrait de Mentha spicata (a) et de Mentha piperita (b).

Mortality of Callosobruchus maculatus according to the doses of extracts of Mentha spicata (a) and Mentha piperita (b). 
Tableau 1 : TL50 (jours) déterminés à partir des différentes doses de poudres et d'extraits.

LT50 (days) determined from the different doses of powders and extracts.

\begin{tabular}{|c|c|c|c|c|c|}
\hline \multirow{2}{*}{ Traitements } & \multirow{2}{*}{ Doses } & \multicolumn{4}{|c|}{ Valeurs des TL50 selon les espèces végétales } \\
\hline & & O. canum & O. gratissimum & M. spicata & M. piperita \\
\hline \multirow{4}{*}{ Poudres $(\mathrm{mg} / \mathrm{g})$} & 10 & 2,8 & 2,8 & 2,5 & 1,8 \\
\hline & 20 & 2,5 & 2,5 & 2,2 & 1,7 \\
\hline & 30 & 2,5 & 2,2 & 2,0 & 1,5 \\
\hline & 40 & 2 & 2,2 & 2,0 & 1,5 \\
\hline \multirow{4}{*}{ Extrait $(\mu 1 / g)$} & 1 & 2,7 & 2,5 & 2,0 & 2,0 \\
\hline & 2 & 2,4 & 2,3 & 1,8 & 1,2 \\
\hline & 3 & 1,9 & 1,9 & 1,1 & 0,8 \\
\hline & 4 & 1,7 & 1,5 & 0,9 & 0,5 \\
\hline
\end{tabular}

Tableau 2 : Effet des traitements sur le taux d'infestation des graines (\%) par C. maculatus.

Effect of treatments on the infestation rate of seed (\%) by C. maculatus.

\begin{tabular}{lccccc}
\hline \multirow{2}{*}{ Traitements } & \multirow{2}{*}{ Doses } & \multicolumn{4}{c}{ Taux d'infestation selon les espèces végétales } \\
\cline { 3 - 6 } & & O. canum $(\mathrm{c})$ & O.gratissimum $(\mathrm{c})$ & M. spicata $(\mathrm{b})$ & M.piperita $(\mathrm{a})$ \\
\hline \multirow{3}{*}{ Poudres $(\mathrm{mg} / \mathrm{g})$} & $10^{*}$ & $87 \pm 2,16(13)$ & $87 \pm 2,16((13)$ & $87 \pm 2,16(13)$ & $84 \pm 2,94(16)$ \\
& $20^{*}$ & $81 \pm 3,8(19)$ & $78,66 \pm 0,94(21,34)$ & $77 \pm 1,41(23)$ & $73 \pm 1,63(27)$ \\
& $30^{*}$ & $69 \pm 2,8(31)$ & $63 \pm 2,16(37)$ & $58,33 \pm 1,88(41,67)$ & $64 \pm 2,94(36)$ \\
& $40^{*}$ & $61 \pm 2,6(39)$ & $58,33 \pm 2,35(41,67)$ & $52,33 \pm 1,24(47,67)$ & $51,33 \pm 0,94(48,67)$ \\
\hline \multirow{2}{*}{ Extrait $(\mu 1 / \mathrm{g})$} & $1^{*}$ & $72 \pm 1,6(28)$ & $79,66 \pm 0,47(20,34)$ & $64,33 \pm 3,29(35,67)$ & $83,33 \pm 1,88(16,67)$ \\
& $2^{* *}$ & $52 \pm 4,2(48)$ & $66 \pm 2,44(34)$ & $48 \pm 2,16(52)$ & $37 \pm 2,16(63)$ \\
& $3^{* * *}$ & $37,66 \pm 2,05(62,34)$ & $30,33 \pm 1,24(69,67)$ & $25,66 \pm 2,05(74,34)$ & $15 \pm 1,63(85)$ \\
& $4^{* * *}$ & $12,66 \pm 2,05(87,34)$ & $10,66 \pm 0,94(89,34)$ & $7,33 \pm 5,24(92,67)$ & $2 \pm 2,8(98)$ \\
\hline
\end{tabular}

( ) : taux de réduction / reduction rate

(a) : espèces à efficacité élevée, (b) : espèces à efficacité intermédiaire, (c) : espèces à efficacité faible

(a) : High efficiency species, (b): medium efficiency species, (c): less efficiency species

${ }^{*}$ : dose de faible efficacité, ${ }^{* *}$ : dose d'efficacité moyenne, ${ }^{* * *}$ : dose de forte efficacité

*: High efficiency dose, ${ }^{* *}$ : medium efficiency dose, ${ }^{* * *}$ : less efficiency dose

\section{Effet des traitements sur la fécondité de C. maculatus}

Les traitements aux poudres comme aux extraits totaux ont provoqué la baisse de la fécondité par rapport au témoin (60,93 \pm 1 oeufs) quelle que soit la dose utilisée. Les plus faibles valeurs de la fécondité ont été obtenues avec les doses les plus élevées. Ainsi, pour les poudres, à la dose de $40 \mathrm{mg} / \mathrm{g}$, le nombre d'œufs pondus par femelle a varié de 10,2 $\pm 0,1$ (O. canum) à 6,6 $\pm 0,16$ (M. piperita); soit les taux de réduction respectifs de 83,25 et $89,16 \%$. Avec les extraits totaux, cette diminution a été beaucoup plus significative. A la plus faible dose $(1 \mu \mathrm{l} / \mathrm{g})$, les valeurs se sont situées entre 10,2 $\pm 0,2$ œufs par femelle ( 0 . gratissimum) et 7,7 $\pm 0,1$ œufs par femelle ( $M$. piperita) ; les taux de réduction s'étant élevés respectivement à 83,25 et 87,36\%. A la dose la plus élevée $(4 \mu \mathrm{l} / \mathrm{l})$ ces taux de réductions ont tendu vers $100 \%: 98,03 \%$ (O. canum et 0 . gratissimum), 98,35 \% (M. spicata) et 99,67\% (M. piperita) ; la fécondité étant quasiment nulle pour les quatre espèces de plante (Tableau 3 ).

\section{Effet des traitements sur l'émergence des adultes de $C$. maculatus}

Le taux d'émergence est le rapport, exprimé en pourcentage, du nombre d'insectes émergés au nombre d'œufs pondus par femelle. Pour 30 femelles, le taux moyen d'émergence a été de $68,6 \pm 1,87 \%$. Cette proportion a diminué considérablement avec les traitements. Ainsi, pour les poudres (toutes doses confondues), les valeurs obtenues varient de 48,2 $\pm 2,6$ à 21,1 $\pm 1,2 \%$; les plus faibles étant enregistrées avec M. spicata $(21,2 \pm 1,9 \%)$ à la dose de $40 \mathrm{mg} / \mathrm{g}$ et $M$. piperita $(21,12 \pm 1,2 \%)$ à celle de 
$30 \mathrm{mg} / \mathrm{g}$. Quant au traitement avec les extraits totaux, il a permis d'obtenir des taux d'émergence dont la valeur la plus élevée $(67,6 \pm 2,7 \%)$ et la plus faible $(5,52 \pm 7,8 \%)$ ont été enregistrées, avec la même plante $M$. pipérita aux doses respectives de $1 \mu \mathrm{l} / \mathrm{g}$ et de $4 \mu \mathrm{l} / \mathrm{g}$.

\section{TRAITEMENT DES GRAINES APRES INFESTATION}

\section{Effet des traitements des œufs et des larves de $C$. maculatus sur l'émergence de l'insecte adulte}

Le taux d'émergence de $C$. maculatus, obtenu à partir des œufs âgés de 0 à 2 jours, a été nul lorsque les traitements des graines ont été faits avec les poudres et les extraits totaux des trois plantes ( $O$. gratissimum, $M$. spicata et $M$. piperita). Ceux obtenus avec $O$. canum ont donné une valeur de $0,5 \%$. Pour les œufs de 3 jours, cette valeur a été de $0,5 \%$, quelle que soit l'origine de la poudre utilisée. Quant aux extraits totaux, ils ont enregistré un taux d'émergence de $1 \%$ à la suite du traitement des graines avec $O$. canum et $O$. gratissimum. Ceux obtenus avec $M$. spicata et $M$. piperita ont été nuls (Tableau 4). En absence de traitement, le taux d'émergence a été de $64 \%$.
Chez les larves, les traitements aux poudres ont fait apparaître 4 classes de taux d'émergence : de 51 à $63 \%$ pour les graines hébergeant les larves de $1^{\text {er }}$ et $2^{\mathrm{e}}$ stade; de 44 à $49 \%$ pour celles renfermant les larves de $3^{\mathrm{e}}$ stade ; de 33 à $38 \%$ pour les graines contenant des larves de $4^{\mathrm{e}}$ stade et de 17 à $19 \%$ pour les nymphes. Les taux d'émergence enregistrés après l'application des extraits totaux se sont répartis également en 3 classes : de 50 à $58 \%$ pour les graines renfermant des larves de $1^{\mathrm{er}}, 2^{\mathrm{e}}$ et $3^{\mathrm{e}}$ stades; de 38 à $42 \%$ pour les graines hébergeant des larves de 4 stade et de 24 à $27 \%$ pour les graines renfermant des nymphes. (Tableau 5).

Le test de Student-Newman-Keuls a permis de faire des classements selon les sources de variations. Deux classes se sont dégagées suivant le type de traitement; celle des extraits et celle des poudres. Les résultats obtenus avec les extraits et les poudres ont été très significativement différents $(P=0,0001)$. II en ressort le classement des espèces végétales dans l'ordre d'efficacité suivant : $M$. piperita, $M$. spicata, $O$. canum et $O$. gratissimum. Quant aux doses, elles se sont regroupées en 3 classes : la 1 1ere comprend toutes les doses de poudre et la plus faible dose des extraits ( 10 , $20,30 \mathrm{mg} / \mathrm{g}, 40 \mathrm{mg} / \mathrm{g}$ pour les poudres et $1 \mu \mathrm{l} / \mathrm{g}$ pour les extraits extraits) ; la $2^{\mathrm{e}}$ est constituée par la dose de $2 \mu \mathrm{l} / \mathrm{g}$ et la $3^{\mathrm{e}}$ regroupe les doses de $3 \mu \mathrm{l} / \mathrm{g}$ et $4 \mu \mathrm{l} / \mathrm{g}$ pour les extraits.

Tableau 3 : Effet des traitements sur la fécondité (nb d'œufs / femelles) de C. maculatus.

Effect of treatments on the fecundity ( $\mathrm{nb}$ of egg / female) of C. maculatus.

\begin{tabular}{lccccc}
\hline \multirow{2}{*}{ Traitements } & \multirow{2}{*}{ Doses } & \multicolumn{4}{c}{ Nombre d'œufs/femelle selon les espèces végétales } \\
\cline { 2 - 6 } & & O. canum (c) & O. gratissimum (c) & M. spicata (b) & M. piperita (a) \\
\hline \multirow{3}{*}{ Poudres $(\mathrm{mg} / \mathrm{g})$} & $10^{*}$ & $17,4 \pm 0,6(71,44)$ & $17,2 \pm 0,2(71,77)$ & $16,03 \pm 0,4(73,69)$ & $16,06(73,64)$ \\
& $20^{*}$ & $12,7 \pm 0,2(79,15)$ & $14,26 \pm 0,1(76,59)$ & $12,8 \pm 0,2(78,99)$ & $14,76 \pm 0,2(75,77)$ \\
& $30^{*}$ & $11,5 \pm 0,2(81,12)$ & $10,13 \pm 0,1(83,37)$ & $8,1 \pm 0,4(86,70)$ & $9,13 \pm 0,1(85,01)$ \\
& $40^{*}$ & $10,2 \pm 0,1(83,25)$ & $9,7 \pm 0,83(84,08)$ & $7,4 \pm 1,2(87,85)$ & $6,6 \pm 0,16(89,16)$ \\
\hline \multirow{2}{*}{ Extrait $(\mu \mathrm{l} / \mathrm{g})$} & $1^{*}$ & $9,5 \pm 0,8(84,40)$ & $10,2 \pm 0,2(83,25)$ & $8,3 \pm 0,3(86,37)$ & $7,7 \pm 0,1(87,36)$ \\
& $2^{* *}$ & $7,36 \pm 0,3(87,92)$ & $7,96 \pm 0,1(86,93)$ & $7 \pm 0,3(88,51)$ & $6,56 \pm 0,2(89)$, \\
& $3^{* * *}$ & $4,13 \pm 0,1(93,22)$ & $4,26 \pm 0(93,00)$ & $4,4 \pm 0,3(92,77)$ & $2,46 \pm 0,1(95,96)$ \\
& $4^{* * *}$ & $1,2 \pm 0,2(98,03)$ & $1,2 \pm 0,2(98,03)$ & $1 \pm 0,8(98,35)$ & $0,2 \pm 0,2(99,67)$ \\
\hline
\end{tabular}

( ) : taux de réduction / reduction rate

(a) : espèces à efficacité élevée, (b) : espèces à efficacité intermédiaire, (c) : espèces à efficacité faible

(a) : High efficiency species, (b): medium efficiency species, (c): less efficiency species

* : dose de faible efficacité, ${ }^{* *}:$ dose d'efficacité moyenne, ${ }^{* *}:$ dose de forte efficacité

*: High efficiency dose, ${ }^{* *}$ : medium efficiency dose, ${ }^{* * *}$ : less efficiency dose 
Tableau 4 : Effet des traitements sur le taux d'émergence (\%) de C. maculatus.

Effect of treatments on the emergency rate (\%) of $\mathrm{C}$. maculatus.

\begin{tabular}{|c|c|c|c|c|c|}
\hline \multirow{2}{*}{ Traitements } & \multirow{2}{*}{ Doses } & \multicolumn{4}{|c|}{ Taux d'émergence selon les espèces végétales } \\
\hline & & O. canum (c) & O. gratissimum (c) & M. spicata (b) & M. piperita (a) \\
\hline \multirow{4}{*}{ Poudres (mg/g) } & $10^{*}$ & $48,2 \pm 2,6(29,74)$ & $47,1 \pm 1(31,3)$ & $38 \pm 1(44,61)$ & $36,5 \pm 1,7(46,79)$ \\
\hline & $20^{*}$ & $44,8 \pm 3,4(34,69)$ & $48,3 \pm 0,5(29,59)$ & $33,3 \pm 2(51,46)$ & $33,5 \pm 0,7(51,17)$ \\
\hline & $30^{*}$ & $37,9 \pm 3,7(44,75)$ & $44,7 \pm 0,1(34,84)$ & $24,6 \pm 0,6(64,14)$ & $21,1 \pm 1,2(69,24)$ \\
\hline & $40^{*}$ & $46,3 \pm 2,4(32,51)$ & $44,6 \pm 2(34,99)$ & $21,2 \pm 1,9(69,10)$ & $24,9 \pm 2,7(63,70)$ \\
\hline \multirow{4}{*}{ Extrait $(\mu 1 / g)$} & $1 *$ & $65,3 \pm 3(4,81)$ & $61,7 \pm 3,3(10,06)$ & $61,7 \pm 2,8(10,06)$ & $67,6 \pm 2,7(1,46)$ \\
\hline & $2^{* *}$ & $53,4 \pm 1,8(22,16)$ & $40,5 \pm 2,2(40,96)$ & $43,8 \pm 1,4(36,15)$ & $40 \pm 3,7(41,69)$ \\
\hline & $3 * * *$ & $51,1 \pm 1,1(25,51)$ & $37,4 \pm 3,4(45,48)$ & $25,9 \pm 2,9(62,24)$ & $25,6 \pm 4,5(62,68)$ \\
\hline & $4 * * *$ & $37,2 \pm 2,8(45,77)$ & $24,4 \pm 4,5(64,43)$ & $25,5 \pm 18,2(62,83)$ & $5,5 \pm 7,8(91,98)$ \\
\hline
\end{tabular}

( ) : taux de réduction / reduction rate

(a) : espèces à efficacité élevée, (b) : espèces à efficacité intermédiaire, (c) : espèces à efficacité faible

(a) : High efficiency species, (b): medium efficiency species, (c): less efficiency species

${ }^{*}:$ dose de faible efficacité, ${ }^{* *}:$ dose d'efficacité moyenne, ${ }^{* *}:$ dose de forte efficacité

*: High efficiency dose, ${ }^{* *}$ : medium efficiency dose, ${ }^{* *}$ : less efficiency dose

Tableau 5 : Effets de poudres $(40 \mathrm{mg} / \mathrm{g})$ et d'extraits $(4 \mu \mathrm{l} / \mathrm{g})$ de quatre espèces végétales sur l'émergence $(\%)$ de $C$. maculatus après traitement des œufs, larves et nymphes.

Effects of powers $(40 \mathrm{mg} / \mathrm{g}$ ) and extracts $(4 \mathrm{\mu l} / \mathrm{g})$ from four vegetable species on the emergency of $\mathrm{C}$. maculatus after eggs, larvae and pupa treatment.

\begin{tabular}{|c|c|c|c|c|c|c|c|c|c|}
\hline \multirow{3}{*}{\multicolumn{2}{|c|}{ Stades de développement }} & \multicolumn{8}{|c|}{ Taux d'émergence selon les espèces végétales } \\
\hline & & \multicolumn{2}{|c|}{ O. canum } & \multicolumn{2}{|c|}{ O.gratissimum } & \multicolumn{2}{|c|}{ M. spicata } & \multicolumn{2}{|c|}{ M. piperita } \\
\hline & & poudre & extrait & poudre & extrait & poudre & extrait & poudre & extrait \\
\hline \multirow{4}{*}{ Eufs } & 0 jour & 0 & 0 & 0 & 0 & 0 & 0 & 0 & 0 \\
\hline & 1 jour & 0 & 0 & 0 & 0 & 0 & 0 & 0 & 0 \\
\hline & 2 jours & 0,5 & 0,5 & 0 & 0 & 0 & 0 & 0 & 0 \\
\hline & 3 jours & 0,5 & 1 & 0,5 & 1 & 0,5 & 0 & 0,5 & 0 \\
\hline \multirow{4}{*}{ Larves } & L1 & 51 & 58 & 62 & 51 & 54 & 55 & 52 & 51 \\
\hline & L2 & 54 & 56 & 63 & 54 & 56 & 57 & 61 & 50 \\
\hline & L3 & 45 & 52 & 49 & 50 & 48 & 54 & 44 & 51 \\
\hline & L4 & 38 & 42 & 33 & 38 & 33 & 40 & 34 & 41 \\
\hline Nymph & & 18 & 24 & 19 & 25 & 17 & 27 & 17 & 25 \\
\hline
\end{tabular}

\section{DISCUSSION}

Les poudres et les extraits totaux des quatre espèces de plante ont réduit l'infestation des graines, la durée de vie, la fécondation et le taux d'émergence de C. maculatus. Cette diminution a été beaucoup plus importante aux fortes doses. Le type de traitement le plus efficace a été celui des extraits totaux pour lequel les valeurs des paramètres étudiés ont été très faibles ou tendant à s'annuler. Les résultats enregistrés ont été comparables à ceux de Dabiré (1993), Nuto (1995) et Kétoh (1998) dont les travaux ont montré que certaines espèces de plantes réduisent la durée de vie des adultes et la fécondité de la femelle de C. maculatus. Ils rejoignent également ceux de Gakuru et Foua-Bi (1995) qui rapportent que les essences végétales sont très efficaces et réduisent la durée de vie de C. maculatus et de Sitophilus orizae. Ainsi, la baisse de la fécondité de l'insecte serait due à la mort précoce causée par l'intoxication respiratoire liée aux composés 
volatils contenus dans les poudres et les extraits totaux (Schmidt et al., 1991; Koumaglo et al., 1996 ; Glitho et al., 1997 ; Mazibur et Gerhard, 1999 et Séri-Kouassi, 2004). Sur les stades préimaginaux, les extraits de chacune des quatre plantes utilisées ont semblé avoir un effet ovicide. Aussi, l'émergence des insectes, issus des graines ayant reçu les traitements au stade nymphal, est-elle intervenue 2 à 3 j après les tests. A cette période, l'effet du traitement a persisté de sorte que les premiers adultes qui ont émergé sont morts. Les autres, nouvellement émergés, ont fui les effets des différents produits et se sont réfugiés dans les loges ayant servi à la nymphose. Les extraits de plantes utilisées seraient donc répulsifs pour les adultes de $C$. maculatus. Ces résultats se rapprochent de ceux de Motto (1995) dont les travaux ont rapporté que les huiles végétales ont un effet toxique sur les embryons contenus dans les œufs des bruches. Cette toxicité serait attribuée à l'effet nocif de certaines substances chémochimiques contenues dans les substances naturelles. En effet, certains composés présents dans les substances naturelles, notamment les terpènes, sont responsables de l'activité insecticide. Cette argumentation est en accord avec celle de SériKouassi (2004) dont les travaux ont montré que les activités ovicide et larvicide des huiles essentielles extraites de certaines plantes est liée à leur richesse en terpène. Ainsi, l'efficacité de Mentha serait due à sa richesse en Menthol (composé majoritaire) tandis que le composé majoritaire de Ocimum est le thymol. Ces composés terpéniques joueraient un rôle répulsif à faible concentration et létal à forte concentration. Ils agissent aussi bien sur le système nerveux que sur la synthèse des protéines chez les insectes (Picimbon, 2002). Aussi, Les trois premiers stades larvaires ( $L 1$, $L 2$, et $L 3$ ) ont semblé moins sensibles aux traitements que le stade $L 4$ et le stade nymphal. Les résultats obtenus après application de 40 $\mathrm{mg}$ de poudre /g de graines de niébé et de $4 \mu \mathrm{l}$ d'extrait /g de graines de niébé, ont montré que le développement des larves n'est pas affecté par les essais. Les différents traitements n'ont pas influencé les larves et les nymphes car les produits n'ont pas traversé le tégument de la graine et par conséquent ne sont pas entré en contact avec les stades préimaginaux de l'insecte. Le tégument constituerait donc une barrière physique à la pénétration des produits dans la graine. Ce constat est également celui de certains auteurs dont les résultats des travaux ont montré que le tégument et les cotylédons des graines de niébé constituent une barrière mécanique qui freine la pénétration des vapeurs des huiles essentielles à l'intérieur des graines, protégeant ainsi les larves (Huignard et al., 2002).

\section{CONCLUSION}

Les poudres et les extraits totaux des quatre espèces de plantes (O. canum, O. gratissimum, $M$. spicata et $M$. piperita) ont montré leur efficacité sur la durée de vie de C. maculatus. Ces traitements ont réduit l'infestation des graines, la fécondité et le taux d'émergence de l'insecte, élevé sur des graines traitées, comparativement au témoin. Les différents essais ont révélé que les extraits totaux des espèces du genre Mentha, notamment $M$. piperita, ont une action certaine dans le contrôle de C. maculatus. Les œufs et les adultes (formes exposées) de C. maculatus ont été plus sensibles aux traitements que les larves et les nymphes (formes cachées). La lutte, au moyen des produits issus de plantes aromatiques, dans les stocks, limiterait les actions déprédatrices des insectes ravageurs et assurerait aux populations consommatrices de niébé, une autosuffisance alimentaire. L'utilisation de pesticides naturels ou biopesticides dans la conservation des denrées alimentaires constituerait une solution dans la résolution des problèmes liés à la protection post-récolte en Afrique, en général, et en Côte d'Ivoire en particulier. Ces insecticides naturels seraient, pour les paysans, une solution plus adaptée par rapport à l'utilisation des insecticides de synthèse, car ces biopesticides sont à faibles coûts et moins toxiques pour l'homme et les animaux.

\section{REFERENCES}

Aboua (L. R. N.). 1997. Etude de quelques aspects du comportement et de la biologie de deux parasites des bruches ravageuses des stocks du Niébé. Mémoire de D.E.A., Université de Cocody, $75 \mathrm{p}$

Alzouma (I.) et (A.) Boubacar. 1987. Effet des feuilles vertes de Boscia senegalensis (Capparidacae) sur la biologie de Bruchidius atrolineatus et Callosobruchus maculatus (Coleoptera-Bruchidae) ravageur des graines de Niébé Vigna unguiculata (L.) WALP In : Actes du colloque 
sur les légumineuses alimentaires en Afrique, Niamey (Niger), 22-25 Nov 1985, AUPELF-UREF ed, 288 - 295.

Caswell (G. H.). 1961. The infestation of cowpeas in the western region of Nigeria. Trop. Sc., $3: 154-158$

Dabire (C.). 1993. Méthodes traditionnelles de conservation du niébé (Vigna unguiculata) au Burkina-Faso. In : Thiam (A.) et Ducom (G.) (éditeur) Protection naturelle des végétaux en Afrique. Dakar : Enda, 45 - 56.

Delobel (A.) et (M.) Tran. 1993. Les Coléoptères des denrées alimentaires entreposées dans les régions chaudes ORSTOM/CTA Paris, 299 - 333

Doumbia (V.). 1992. Influence de la nourriture sur quelques éléments de la biologie de Callosobruchus maculatus FAB (Coloeptera-Bruchidae). Mémoire de D.E.A., Université d'Abidjan, 46 p.

Foua-Bi (K.). 1993. Produits naturels utilisés dans la prévention des stocks en Afrique noire. In : Thiam (A.) et Ducom (G.) (éditeur) Protection naturelle des végétaux en Afrique. Dakar : Enda, 85 - 100.

Gakuru (S.) et (K.) Foua-Bi. 1995. Effet comparé des huiles essentielles de quatre espèces végétales contre la bruche du niébé (Callosobruchus maculatus FAB) et le charançon du riz (Sitophilus oryzae L). Agriculture, 13 (4) : 143 - 146.

Glitho (I. A.), (K. G.) Ketho et (H. K.) Koumaglo. 1997. Effets de quelques huiles essentielles sur l'activité reproductrice de Callosobruchus maculatus Fab. "Annales de l'Université de Ouagadougou" Série B, $5: 174-185$.

Huignard (J.), Gugravot (S.), Ketho (K. G.), Thibout (E.) et (I. A.) Glitho. 2002. Utilisation de composés secondaires de végétaux pour la protection des graines d'une légumineuse, le niébé. Conséquences sur les insectes ravageurs et leurs parasitoïdes. In : Biopesticides d'origine végétale, 133 - 149.

Ketho (K. G.). 1998. Utilisation des huiles essentielles de quelques plantes aromatiques du Togo comme biopesticides dans la gestion des stades de développement de Callosobruchus maculatus F. (Coleoptera : Bruchidae). Thèse de Doctorat Univ. Du Bénin LoméTogo, $136 \mathrm{p}$.

Koumaglo (K. H.), (K.) Akpagana, Glitho (I. A.), (F. X.) Garneau, Gagnon (H.) and (F. I.) Jean. 1996. Germinal and Neral, Major constituents of Lipia multiflora Moldenke leaf oil J. Essent. Oil Res., 8 : 237 - 240
Kossou (D. K.) et (N.) Aho. 1993. Stockage et conservation des grains alimentaires tropicaux, principes et pratiques. Edit Flamboyant, Cotonou, $125 \mathrm{p}$.

Mazibur (M. R.) et Gerhard (H. S.). 1999. Effect of Acorus calamus (L) (Araceae) essential oil papours from various origins on Callosobruchus phaseoli (Gyllenhal) (Coleoptera : Bruchidae) J.stored - ProdRes.,35 : 285 - 295.

Motto (O. C.). 1995. Effet des variétés de niébé Vigna unguiculata (L) WALP et des huiles végétales sur le développement de Callosobruchus chinensis (L) BRIDWELL. Mémoire de D.E.A. Université de Dschang, $41 \mathrm{p}$.

Nuto (Y.). 1995. Synergic action of co-occuring toxin in root barkof Zanthoxylum zanthoxyloides (Routaceae) against the cowpea bettle Callosobruchus maculatus (Coleoptera : Bruchidae). Thesis of PhD. DS.U.N.Y. Syracuse, New York, 107 p.

Nuto (Y.) et (A.) Glitho. 1990. Des rapports entre les bruchidae déprédateurs des légumineuses alimentaires cultivées au Togo et leurs plantes-hôtes - Actes J.sci. Univ.du Benin. Presse UB/Lomé, 2 : 312 - 325

Ostermann (H.). 1993. Utilisation des insecticides au Sahel. In : Thiam et Ducom : Protection naturelle des végétaux en Afrique-Dakar. Enda, 115 - 132.

Picimbon (J. F.). 2002. Protéines liant les odeurs (OBP) et protéines chimiosensorielles (CSP) : cibles moléculaires de lutte intégrée. In : Biopesticides d'origine végétale. Ed. TEC et DOC, Paris, 265 - 283

Rachie (K. O.) and (L. M.) Roberts. 1974. Grain legumes of lowland tropic. Advances In : Agronomy, $26: 44-46$.

Seri-Kouassi (B.), and PH. 2004. Entomofaune du niébé (Vigna unguiculata L WALP) et impact des huiles essentielles extraites de neuf plantes locales sur le reproduction de Callosobruchus maculatus FAB (Coleoptera : Bruchidae) en Côte d'lvoire. Thèse de doctorat d'Etat ès sciences, spécialité entomologie, Université de Cocody, 199 p.

Schmidt (G. H.), Risha (E. M.) and (A. K. W.) ElNahal. 1991. Reduction of progeny of some stored-product Coleoptera by vapours of Acorus calamus. J. stored Pro. Res., 27 (2) : $121-127$

Stanton (R. W.). 1970. Les légumineuses à graines en Afrique. Publ. FAO, 199 p.

Thiam (A.) et (G.) Ducom. 1993. Toxicité et risque d'emploi des pesticides pour la faune sauvage In: Protection naturelle des végétaux, Dakar, Enda Edition, Série Etudes et Recherche, 17 - 36. 